\title{
Distal left main bronchial injury after catheter ablation for atrial fibrillation: Report of two cases
}

\author{
Aaron Sparks, James R Stewart*, Jonathan Kozinn and Alan Wimmer \\ University of Missouri Kansas City School of Medicine, USA
}

\begin{abstract}
We report two patients who developed respiratory complications after catheter ablation for atrial fibrillation seen recently at our institution. The first patient presented with worsening dyspnea. Outpatient bronchoscopy was performed and demonstrated and a pin-point opening in the distal left main bronchus 6 months after $\mathrm{RF}$ and cryothermal ablation. The patient was managed with rigid bronchoscopy, left main bronchial dilatation and silastic stent placement. The second patient presented within 24 hours of the procedure at an outside institution with severe prolonged episodes of cough and dyspnea, and acute respiratory failure. He had pneumopericardium in addition to his respiratory symptoms. Fiberoptic bronchoscopy was performed and the pericardium was drained surgically. He was found to have a full thickness thermal injury with loss of bronchial integrity immediately before the left main bifurcation, along the anterior surface of the bronchus. He was supported with ventilation, serial bronchoscopic evaluations, hemodynamic and antibiotic support, and eventual tracheostomy. Both patients are improved after treatment. We believe these are the first two reported cases of this complication following radiofrequency catheter ablation.
\end{abstract}

\section{Introduction}

Since its introduction in the 1990's, the indications for catheter ablation of the left atrium for atrial fibrillation have widened to include more patients. Certain potential major and minor complications have been recognized [1]. Major complications include periprocedural death $(0.15 \%)$, esophageal injury with leak or aortoesophageal fistula $(0.04 \%)$, cerebral thromboembolic events, TIA and stroke, $(0.94 \%)$, cardiac perforation with hemorrhage and tamponade (1.31\%), pulmonary venous stenosis $(0.29 \%)$, phrenic nerve injury $(0.48 \%)$ and left atrial tachyarrhythmias, specifically left atrial flutter. Minor complications have included femoral false aneurysms or thrombosis $(0.93 \%)$ and arteriovenous fistulas $(0.54 \%)$. Some arterial injuries may lead to retroperitoneal hematoma, and concurrent anticoagulation may play a role.

We present two patients who experienced injury to the distal left main bronchus as a consequence of RF and caryballoon ablation for atrial fibrillation. The first patient presented 6 months after the RFA, which had been performed after a failed cryoablation. He developed progressive onset of dyspnea with minimal exertion. The second patient presented within 24 hours of the RFA with severe coughing paroxysms, dyspnea and respiratory failure. We describe the management of these patients.

\section{Case 1}

The patient is a 67 -year-old man who had symptomatic paroxysmal atrial fibrillation. He failed pharmacologic therapy and cardioversion and underwent left atrial cryoablation. Within 6 weeks his atrial fibrillation had returned. He subsequently underwent a successful $\mathrm{RF}$ ablation with resolution of the atrial fibrillation. Six months later, the patient had developed progressive dyspnea, so severe he could no longer walk on the golf course, and even had trouble getting to and from the green on each hole. He was admitted to hospital and had fiberoptic bronchoscopy demonstrating a pin-point opening in the distal left main bronchus. The patient underwent rigid bronchoscopy, dilatation of the distal left main bronchus and placement of an 8X13 MM Hood silastic stent. He noted immediate improvement in his symptoms. Approximately 3 weeks later during a coughing episode the stent became dislodged and was expectorated. He underwent CT of the chest with $3 \mathrm{D}$ reconstruction of the tracheobronchial tree (Figure 1), which documented the area of stenosis. He had repeat rigid bronchoscopy and dilatation, with placement of a 10X13 mm Hood stent. Patency of the upper and lower lobe bronchi beyond the stent were confirmed, and the patient was discharged home. He had mild residual dyspnea but was greatly improved. He has had no further stent migration. Twelve months after initial bronchoscopy the stent was removed, and his symptoms are minimal.

\section{Case 2}

The patient is a 34-year-old man with symptomatic paroxysmal atrial fibrillation, poorly controlled with medication. He was taken to the electrophysiology laboratory at an outside institution where RFA was performed. The procedure seemed to be uncomplicated. The patient recovered and was discharged home. Within 24 hours he returned to the hospital with prolonged severe coughing episodes, hypoxemia and dyspnea. Chest radiograph demonstrated pneumopericardium. He was intubated for respiratory failure and transferred to our institution, where he was found to be hypotensive, and hypoxemic. After a brief period of stabilization, he was taken to the operating room where in addition to fiberoptic bronchoscopy, he underwent subxiphoid pericardial drainage

*Correspondence to: James R Stewart, University of Missouri Kansas City School of Medicine, Department of Surgery, 2301 Holmes Road, Kansas City, MO 64108, USA, Tel: 816-404-5463; E-mail: jrs1040@aol.com

Key words: atrial fibrillation, bronchial injury and stenosis, bronchial stent, catheter ablation

Received: June 19, 2018; Accepted: June 26, 2018; Published: June 29, 2018 
and placement of a 24 Fr. Jackson-Pratt drain. At initial presentation, there appeared to be dark, slough on the inferior wall of the distal left main bronchus. The patient was treated for respiratory failure and sepsis, and received serial bronchoscopic examinations (Figure 2A-2C). It became clear that the patient had suffered a bronchial to pericardial fistula secondary to the ablation. The integrity of the left atrium remained intact. With continued support the injury showed signs of healing. Although stent placement was considered, it did not appear necessary as his condition improved. Eventually a tracheostomy was placed, and the patient successfully weaned from ventilator support. He was discharged to a rehabilitation facility closer to home but returned 6 weeks later for repeat bronchoscopy and possible downsizing or removal of the tracheostomy tube. Bronchoscopy at that time showed nearly complete healing of the injury with no evidence of stenosis. Tracheostomy was removed.

\section{Discussion}

The patients in this report present different spectra of thermal injury to the distal left main bronchus as a consequence of catheter ablation for atrial fibrillation. The first patient had late bronchial

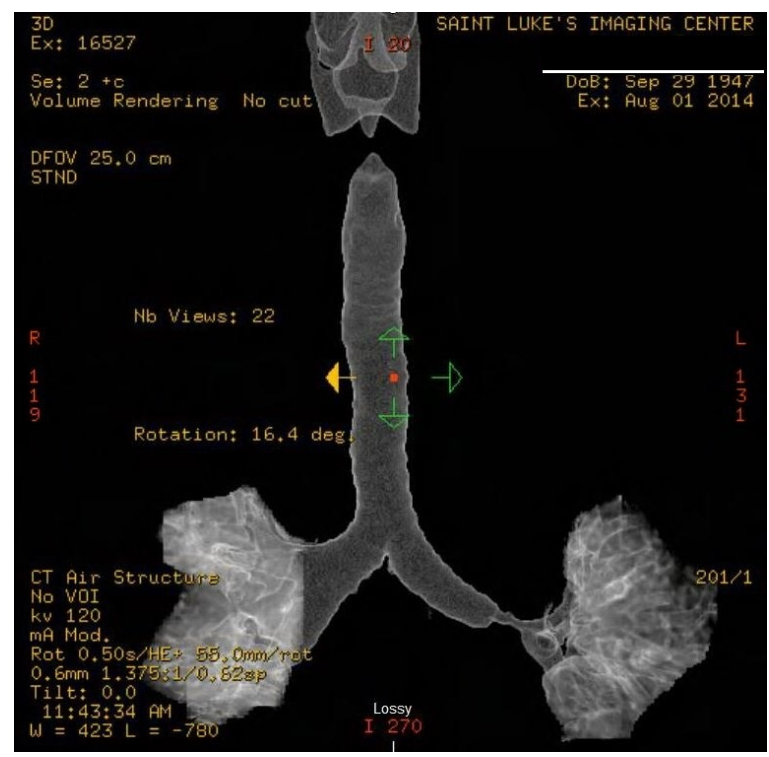

Figure 1. Left main bronchial stenosis after initial dilatation and stent dislodgment. Threedimensional reconstruction of tracheal CT, Case 1

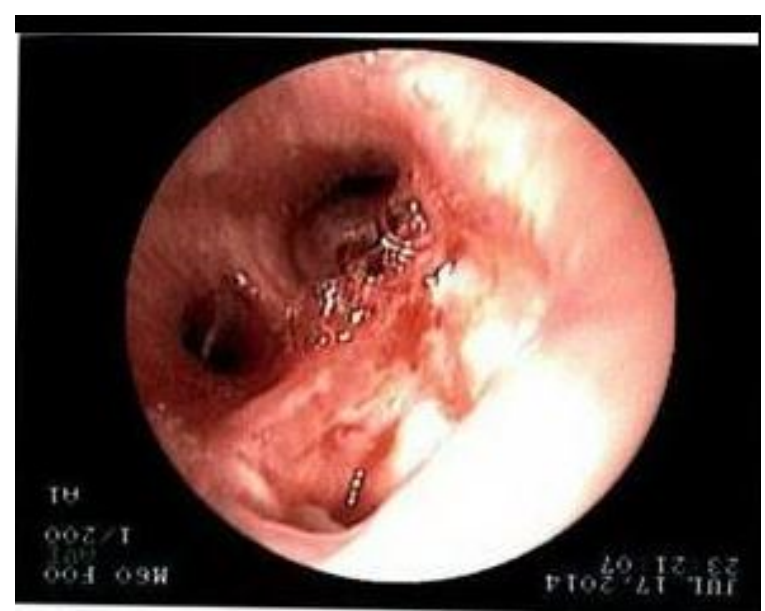

Figure 2A. Bronchoscopy at diagnosis, Case 2

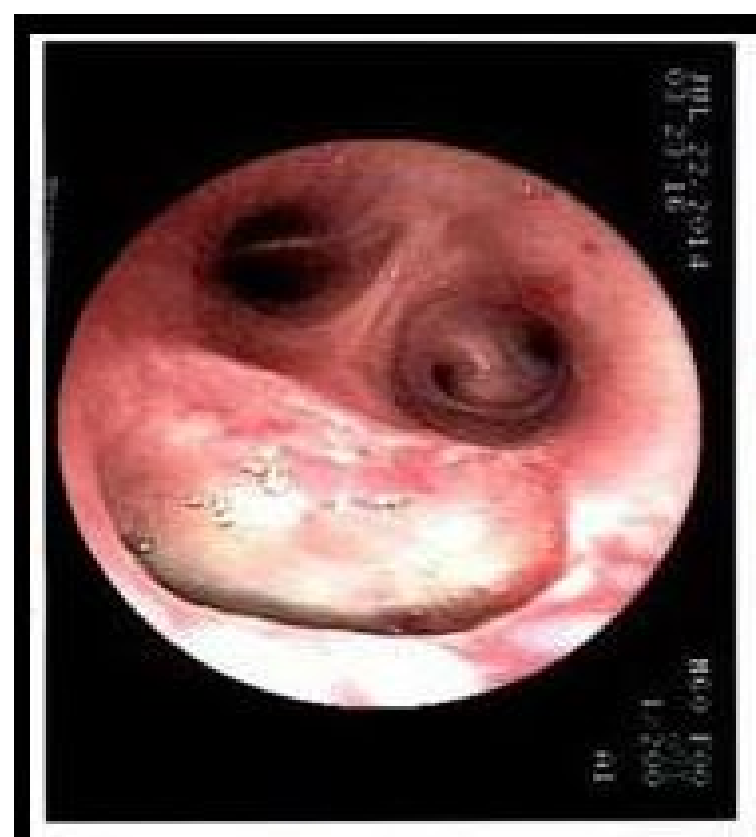

Figure 2B. Bronchoscopy after 11 days, Case 2

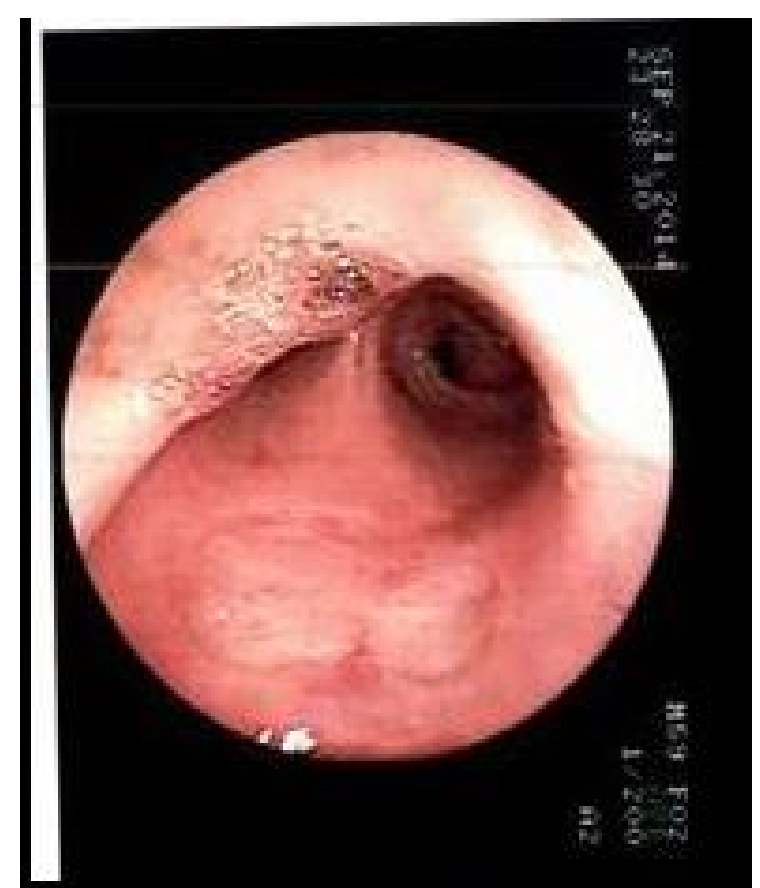

Figure 2C. Bronchoscopy at 60 days, Case 2

stenosis, as demonstrated in the enclosed tracheobronchial 3D CT reconstruction. This study was performed after stent dislodgement and before repeat bronchoscopic dilatation and stent replacement. The patient was evaluated for possible left upper pulmonary venous stenosis, by computed tomographic arteriogram (CTA), and found to have narrowing of the left superior pulmonary vein. The second patient was critically ill with a communication between his bronchus and pericardium, respiratory failure and sepsis, probably as a result of aspiration pneumonia. It is fortunate that this patient did not have hemorrhage due to communication between the pulmonary vein, the bronchus and pericardium. This patient was screened for pulmonary 
venous and bronchial stenosis as a consequence of RFA and has thus far demonstrated no significant narrowing after 9 months.

This complication has been reported after cryballoon ablation and a recent study suggests that ice formation in the left mainstem bronchus during this procedure is common [2-6]. We are unaware of prior reports of this complication following radiofrequency ablation. It is important to keep in mind the proximity of the left atrium and pulmonary veins to important anatomic structures including the esophagus and aorta, distal left main and left upper lobe bronchi, the right pulmonary artery and right and left phrenic nerves. With caution, and greater focus on the surrounding anatomy, it is hoped that these complications can be avoided. If endoscopic therapy had not been helpful in the first patient, an open bronchoplastic procedure may have been necessary. In addition to computer guided ablation systems, and esophageal temperature monitoring, preprocedural computed tomography or magnetic reasonance imaging, or intraprocedural transesophageal or intracardiac echocardiography may be able to direct catheter placement in patients who might be at risk.

\section{References}

1. Sorgente A, Chierchia GB, de Asmundis C, Sarkozy A, Capulzini L, et al. (2011) Complications of atrial fibrillation ablation: when prevention is better than cure. Europace 13: 1526-1532. [Crossref]

2. Van Opstal JM, Timmermans C, Blaauw Y, Pison L (2011) Bronchial erosion and hemoptysis after pulmonary vein isolation by cryoballoon ablation. Heart Rhythm 8: 1459 .

3. Martí-Almor J, Jauregui-Abularach ME, Benito B, Vallès E, Bazan V1, et al. (2014) Pulmonary hemorrhage after cryoballoon ablation for pulmonary vein isolation in the treatment of atrial fibrillation. Chest 145: 156-157. [Crossref]

4. Desai AK, Osahan DS, Undavia MB, Nair GB (2015) Bronchial injury postcryoablation for atrial fibrillation. Ann Am Thorac Soc 12: 1103-1104. [Crossref]

5. Cuoco F, Sturdivant JL, Wharton JM, Gold MR (2016) Delayed formation of an atrial bronchial fistula following cryoballoon ablation for atrial fibrillation. Heart Rhythm 13: S156

6. Verma N, Gillespie CT, Argento AC, Tomson T, Dandamudi S, et. al. (2017) Bronchial effects of cryballoon ablation for atrial fibrillation. Heart Rhythm 14: 12-16. [Crossref]

Copyright: $\odot 2018$ Sparks A. This is an open-access article distributed under the terms of the Creative Commons Attribution License, which permits unrestricted use, distribution, and reproduction in any medium, provided the original author and source are credited. 